\title{
A new technique to prevent curling of guide wire in urinary bladder during J stent insertion with flexible cystoscope
}

\author{
Nasir Mahmood, MD; Tahir Pasha, MD
}

Western Memorial Regional Hospital, Corner Brook, NL, Canada

Cite as: Can Urol Assoc J 2016;10(1-2):E34-5. http://dx.doi.org/10.5489/cuaj.3079

Published online January 14, 2016.

\section{Abstract}

Insertion of J stent is a very common procedure performed by urologists. This procedure is often performed to relieve ureteral obstruction due to stones, tumours, ureteral kink, or stricture on urgent basis. An increasing number of urologists are using a flexible cystoscope and local anaesthetic for J stent insertion. Occasionally, the guide wire curls in the bladder, making it impossible to insert the J stent. Here, we describe a simple method to overcome this problem using modified ureteral dilator, which is usually available in the cystoscopy suite.

\section{Method}

Insertion of J stent was first described to the urological society in 1978 by Finney et al. ${ }^{1}$ Indications for ureteral stent placement include ureteric obstruction due to benign or malignant conditions. ${ }^{2,3}$ The procedure is frequently performed via flexible cystoscope in cysto-suite as an outpatient procedure. ${ }^{4}$ In most cases, the procedure is straightforward; however, occasionally the guide wire loops in the bladder (Fig.1) preventing J stent insertion. There are various ways to overcome this problem, including removing the guide wire and trying again, getting rid of the loop by holding the wire with forceps at the vesicoureteric junction, and straightening the loop or try different types of guide wires. Sometimes none of these methods work, resulting in prolonged operative time or abandoning the procedure.

The next step is to resort to rigid cystoscope. The rigid cystoscope stabilizes the guide wire up to the ureteric orifice, preventing it from curling in the bladder. The use of rigid cystoscope requires changing the patient's position to lithotomy, redraping, asking for rigid scope setup, and may also require sedation or general anesthesia. The rigid cystoscope is less desirable under local anesthesia than flexible cystoscopy, as it is more painful. ${ }^{5}$

We came up with a new idea and used a $16 \mathrm{~F}$ ureteral dilator out of the ureteral dilator set made by Cook (AQ 076000).This is cut at the tip to allow the passage of 5 French J stent and also shortened to a length of $20 \mathrm{~cm}$ from its original length of $60 \mathrm{~cm}$ (Fig. 2). First, the guide wire is inserted via flexible cystoscope up to the ureteric obstruction or kink. This is followed by removal of flexible cystoscopy, leaving the guide wire in place. The modified ureteral dilator is then threaded over the guide wire under fluoroscopy control, with the tip of the dilator positioned at the upper end of the symphysis pubis. The J stent, followed by the pusher, is loaded over the wire. Progress of the bladder end of $J$ stent is monitored by the metallic tip of the pusher, which is visible through the ureteral dilator with fluoroscopy.

As the radio-opaque metallic tip is seen emerging from the ureteric dilator, the guide wire is removed, leaving the $\mathrm{J}$ stent in optimal position. Just like a rigid scope, the modified ureteral dilator stabilizes the guide wire close to the ureteric orifice under fluoroscopy to prevent the looping of wire in the bladder (Fig. 3). To our knowledge this technique has not been published before.

\section{Conclusion}

If the guide wire keeps looping in the bladder during stent insertion with flexible cystoscope, a modified ureteral dilator can be used before proceeding to rigid cystoscopy.

Competing interests: The author declares no competing financial or personal interests.

This paper has been peer-reviewed. 


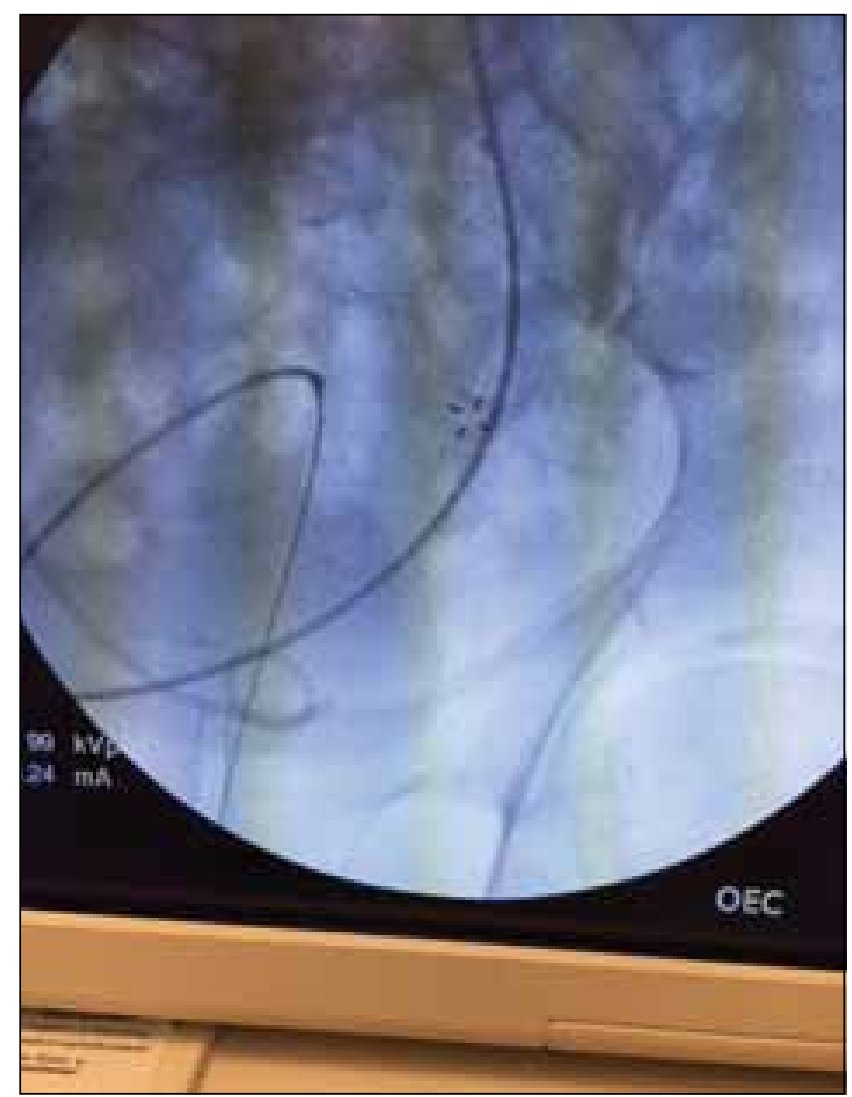

Fig. 1. Guide wire curled in bladder.

\section{References}

1. Hepperlen TW, Mardis HK, Kammendal H. Self-retained internal ureteral stents: A new approach. J Urol 1978;119:731-4.

2. Fiuk J, BaoY, calleary JG, et al. The use of internal stents in chronic ureteral obstruction. J Urol 2015;193:1092-100. http://dx.doi.org/10.1016/i.juro.2014.10.123

3. Sountoulides $P$, Pardalidis $N$, Sofikitis N. Endourologic management of malignant ureteral obstruction: indications, results, and quality-of-life issues. J Endourol 2010;24:129-42. http://dx.doi.org/10.1089/ end.2009.0157

4. Adeyoju $A B$, Collins $G N$, Broomanand P, et al. Outpatient flexible cystoscope-assisted insertion of ureteric catheters and ureteric stents. BJU Int 1999;83:748-50.

5. Seklehner $S$, Remzi $M$, Fajkovic $H$, et al. Prospective multi-institutional study analyzing pain perception of flexible and rigid cystoscopy in men. J Urol 2015;85:737-41. http://dx.doi.org/10.1016/j.urology.2015.01.007

Correspondence: Dr. Nasir Mahmood, Western Memorial Regional Hospital, Corner Brook, NL, Canada; nmnasirmahmood@gmail.com

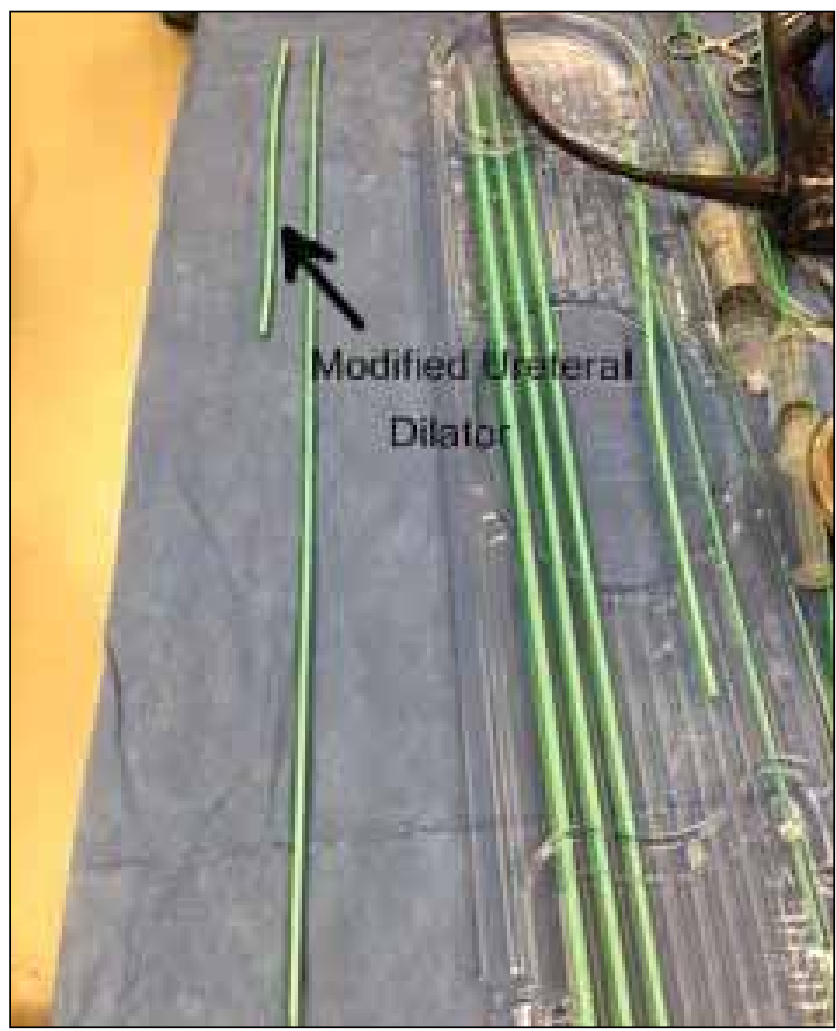

Fig. 2. Modified ureteral dilator prepared from Cook ureteral dilator set.

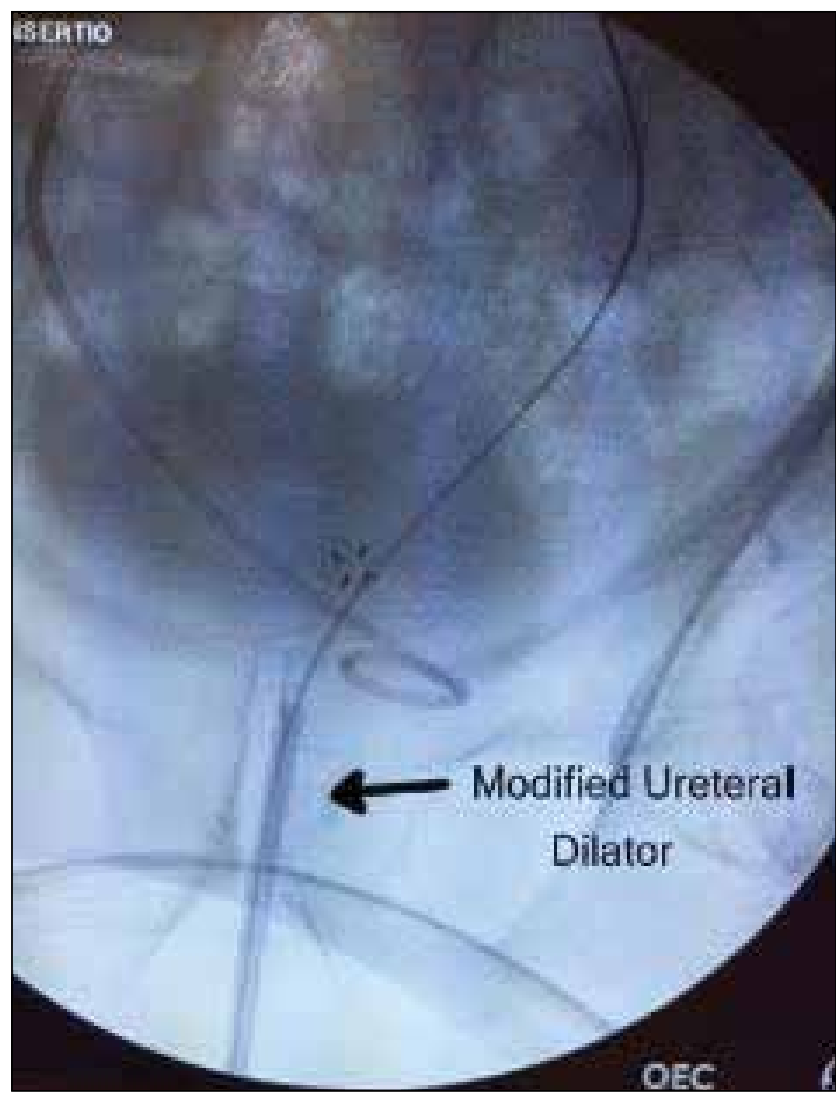

Fig. 3. Modified ureteral dilator used for stent insertion in left ureter. 